\title{
Stratégie de lutte envers une néophyte envahissante - exemple de la puéraire
}

\author{
Romina Morisoli \\ Marco Conedera \\ Giorgio Moretti \\ Simona Crivelli \\ Veronica Soldati \\ Mario Bertossa \\ Gianni Boris Pezzatti
}

\author{
Agroscope Centre de recherche Cadenazzo $(\mathrm{CH})^{*}$ \\ Institut fédéral de recherches sur la forêt, la neige et le paysage $(\mathrm{CH})$ \\ Sezione Forestale Cantonale, Ticino $(\mathrm{CH})$ \\ Institut fédéral de recherches sur la forêt, la neige et le paysage $(\mathrm{CH})$ \\ Institut fédéral de recherches sur la forêt, la neige et le paysage $(\mathrm{CH})$ \\ Agroscope Centre de recherche Cadenazzo $(\mathrm{CH})$ \\ Institut fédéral de recherches sur la forêt, la neige et le paysage $(\mathrm{CH})$
}

\begin{abstract}
Stratégie de lutte envers une néophyte envahissante - exemple de la puéraire
La puéraire (Pueraria lobata) est une liane originaire de l'Asie orientale qui en conditions optimales peut croître jusqu'à $30 \mathrm{~cm}$ par jour. En Suisse, cette espèce figure sur la Liste noire des espèces envahissantes. Actuellement, 41 foyers sont signalés en Suisse italienne pour une totalité de $35141 \mathrm{~m}^{2}$ colonisés. Au cours des six dernières années, le taux d'augmentation en surface des foyers historiques a été de $45 \%$, 16 nouveaux foyers ont été découverts et quatre vieux foyers ont été éradiqués. Les graines produites par les lianes adultes sont en mesure de germer, même à l'état sauvage. La puéraire a donc franchi la phase de naturalisation et elle pourrait bientôt entrer dans un stade d'expansion rapide, ce qui suggère l'adoption d'une stratégie d'éradication totale à moyen terme. Des essais préliminaires ont permis de confirmer l'efficacité des méthodes de lutte chimique et de tester la lutte mécanique (coupe des racines du nœud) pour les applications en forêt et dans les sites protégés. Ce cas d'étude concernant la puéraire est l'exemple idéal d'une utilisation combinée de l'approfondissement des connaissances sur l'écologie de l'espèce et de l'évaluation précoce des méthodes de contrôle. Le but étant le contrôle, voire l'éradication systématique d'une espèce envahissante avec des coûts raisonnables.
\end{abstract}

Keywords: Pueraria lobata, kudzu, invasive neophytes, eradication, control methods doi: $10.3188 /$ szf.2018.0102

*A Ramél 18, CH-6593 Cadenazzo, courriel romina.morisoli@agroscope.admin.ch
$\mathrm{L}$ a globalisation des échanges commerciaux et la circulation des personnes sont les principales responsables de l'introduction d'espèces exotiques envahissantes hors de l'aire de distribution naturelle (Hulme 2009). Les activités de coordination et de lutte directe envers ces espèces sont généralement importantes et les moyens à disposition limités, ce qui suggère un emploi très ciblé des ressources (Bischoff et al 2014). D'après Kueffer et al (2014), les stratégies les plus prometteuses en termes de coûts-efficacité dans la lutte contre les espèces envahissantes sont la prévention, ayant pour but d'empêcher ou de retarder l'arrivée d'une espèce, et la détection précoce de leur présence sur le territoire dans le but d'un confinement, voire d'une éradication rapide.

Le cas de la puéraire hérissée (Pueraria lobata), une espèce inscrite sur la Liste noire des espèces exotiques envahissantes en Suisse (Info Flora 2013), est assez représentatif à ce sujet, tout au moins à l'échelle régionale. Voigt (1920) en fait déjà mention à l'état sauvage lorsqu'il signale deux foyers qui produisent des fleurs et des graines dans les communes de Magliaso et Morcote au sud du Tessin. Il signale l'utilisation de la puéraire par les paysans comme fourrage pour les chèvres et suggère une possible culture de l'espèce sur les escarpements. Le premier inventaire systématique de la puéraire au sud des Alpes suisses, permet à Pron (2006) de répertorier 21 sites. En 2011, une surveillance par réplication du même inventaire a permis la découverte de sites supplémentaires et de confirmer la tendance d'accroissement des surfaces déjà connues en 2006 (+29\%; Morisoli et al 2013).

Le but de cette contribution est de synthétiser les connaissances actuelles concernant la biologie et l'écologie de l'espèce, d'analyser en détail la distribution actuelle, la tendance évolutive et le potentiel d'expansion future de la puéraire au sud des Alpes 
suisses et de tester différentes méthodes de lutte pour son contrôle et son éradication. Cette contribution permet d'esquisser, sur la base des connaissances acquises, une stratégie de gestion et de lutte envers l'espèce à moyen terme.

\section{Etat des connaissances sur la puéraire}

\section{Biologie et écologie}

La puéraire hérissée (appelée aussi vigne du Japon ou Kudzu en anglais) se nomme scientifiquement Pueraria lobata [Willd.] Ohwi (syn. Pueraria montana var. lobata [Willd.] Sanjaooa \& Predeep) en hommage au botaniste suisse Marc Nicolas Puerari (Mitich 2000).

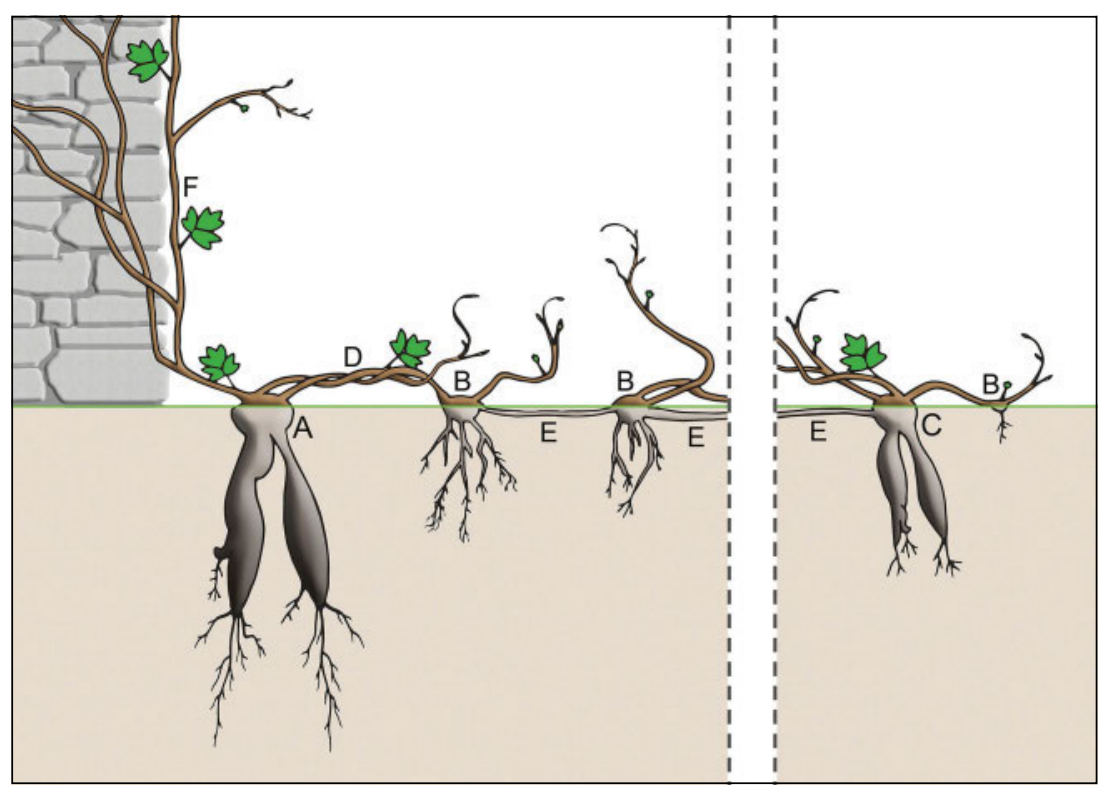

Fig. 1 Visualisation schématique de la morphologie de la puéraire. A) plante mère; $B)$ nœud enraciné; C) nœud enraciné devenu autonome; D) liane à développement horizontal; E) liane enterrée; F) liane à développement vertical. Dessin: Rita Conedera et Patrik Krebs, 2017

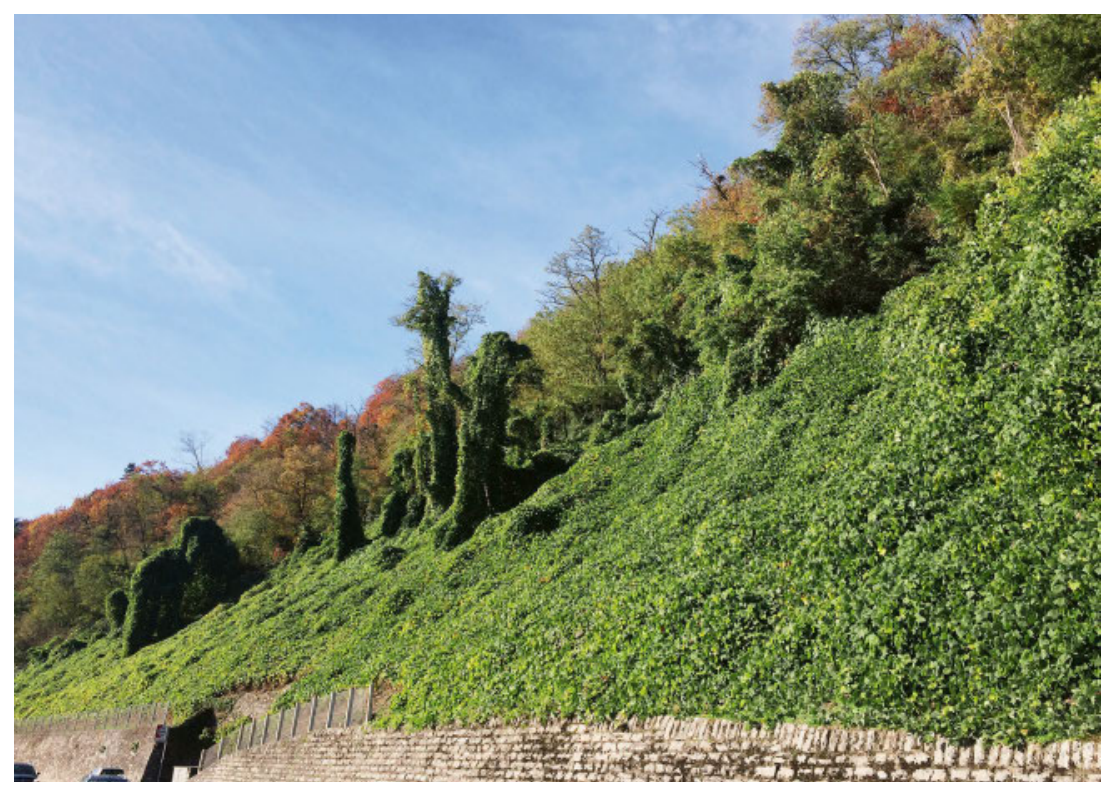

Fig. 2 Vue du foyer historique de Magliaso TI. Photo: Romina Morisoli, 2016
Il s'agit d'une liane de la famille des fabacées (Fabaceae) originaire d'Asie orientale tempérée (Alderman 2004). Les tiges et les racines de cette espèce se développent à partir de nœuds enracinés (collets) à la surface du sol (figure 1). Le système racinaire a la capacité de former de grosses racines tubéreuses de stockage de réserves vitales et d'eau (Mitich 2000) qui peuvent atteindre des grandes dimensions ( $2 \mathrm{~m}$ de long, $30 \mathrm{~cm}$ de diamètre et jusqu'à $180 \mathrm{~kg}$; Alderman 2004) et représenter plus de 50\% de la biomasse de la plante (Invasive Species Specialist Group ISSG 2017). Les nœuds enracinés peuvent produire jusqu'à 25 jeunes tiges herbacées, de couleur vert strié de pourpre et couvertes de poils de couleur bronze. Ces dernières se développent en s'étendant au sol ou en grimpant sur tout objet capable de les supporter (figure 1). En conditions idéales, ces tiges peuvent pousser jusqu'à 20-30 m par an, avec des maxima allant jusqu'à $30 \mathrm{~cm}$ par jour (Mitich 2000, Alderman 2004). Au sud des Alpes suisses, Pron (2006) a mesuré des croissances jusqu'à $26 \mathrm{~cm}$ par jour, avec des lianes poussant jusqu'à 15 m par an. Dans le temps, les nœuds enracinés peuvent même s'affranchir de la plante mère (figure 1). Le résultat d'une telle vigueur et dynamique de croissance est la formation d'un dense réseau de tiges enchevêtrées avec des grandes feuilles vertes caduques, alternes et trifoliolées qui rendent les amas presque impénétrables (figure 2). La reproduction végétative des nœuds est donc un moyen très efficace de propagation sur courte distance (Tsugawa \& Kayama 1985).

Entre juillet et septembre, sur les parties grimpantes exposées au soleil apparaissent des racèmes de 10-20 cm de long avec beaucoup de fleurs de couleur rouge-pourpre qui deviennent des gousses, tandis que sur les lianes fines et à croissance horizontale au niveau du sol, seules des ébauches de fleurs sont produites. La production de graines dans les gousses dépend du microclimat et elle n'est généralement pas énorme (Lindgren et al 2013). Les graines sont rondes et se distinguent par leur coloration entre semis mûrs et immatures et par leur dimension qui varie entre 3 et $4 \mathrm{~mm}$ environ (Mitich 2000). Les graines ont une dormance physique qui doit être levée pour permettre la germination (Susko et al 2001). La biologie des graines de la puéraire est peu connue, même si des essais de germination avec scarification ont démontré des taux de germination assez haut qui peuvent dépasser les 90\% (Lindgren et al 2013). Même si la production de graines viables en mesure de survivre dans le sol au moins une année existe, la reproduction sexuelle ne semble pas être le moyen principale de diffusion de l'espèce. Cela d'autant plus que les graines non charnues de la puéraire ne sont pas spécialement recherchées par les oiseaux, ce qui limite beaucoup l'efficacité de leur dispersion (Meisenburg \& Fox 2002). 
Les connaissances sur les exigences climatiques de l'espèce sont encore très approximatives et doivent être évaluées pour l'Europe. En Amérique, la puéraire est présente dans les régions ayant des précipitations annuelles excédant $1000 \mathrm{~mm}$ et dont la période de végétation est longue et caractérisée par des étés chauds (température du mois le plus chaud $>22-25^{\circ} \mathrm{C}$ ). Les hivers doux (température hivernale moyenne $>5{ }^{\circ} \mathrm{C}$; Mitich 2000) sont aussi favorables à l'espèce, même si sa présence est signalée au Canada où la température du mois le plus froid est de $-4.6^{\circ} \mathrm{C}$ et la température annuelle moyenne de $9.2^{\circ} \mathrm{C}$. Les tiges lignifiées peuvent résister jusqu'à $-27{ }^{\circ} \mathrm{C}$ (Lindgren et al 2013). Follak (2011) définit une température minimale de $12{ }^{\circ} \mathrm{C}$ pour le départ de la saison végétative et une limite supérieure de 35 ${ }^{\circ} \mathrm{C}$ pour le stress de chaleur. En marge de l'optimum climatique, les lianes ne sont plus en mesure de fleurir et deviennent très sensibles au gel précoce. En cas de dégâts de gel, le redémarrage végétatif de la plante est garanti par les nœuds enracinés (Coiner 2012).

\section{Comportement invasif et mesures de contrôle}

Le potentiel envahissant de la puéraire s'est manifesté d'abord au sud-est des Etats-Unis, où sa présence comme plante ornementale était déjà documentée depuis la deuxième moitié du XIX siècle (Lindgren et al 2013). Elle a été ensuite utilisée comme plante fourragère pour le bétail et dans les années 1930, sa plantation a même été encouragée pour le contrôle de l'érosion des sols (Alderman 2004). L'absence de plantes antagonistes, de rava-

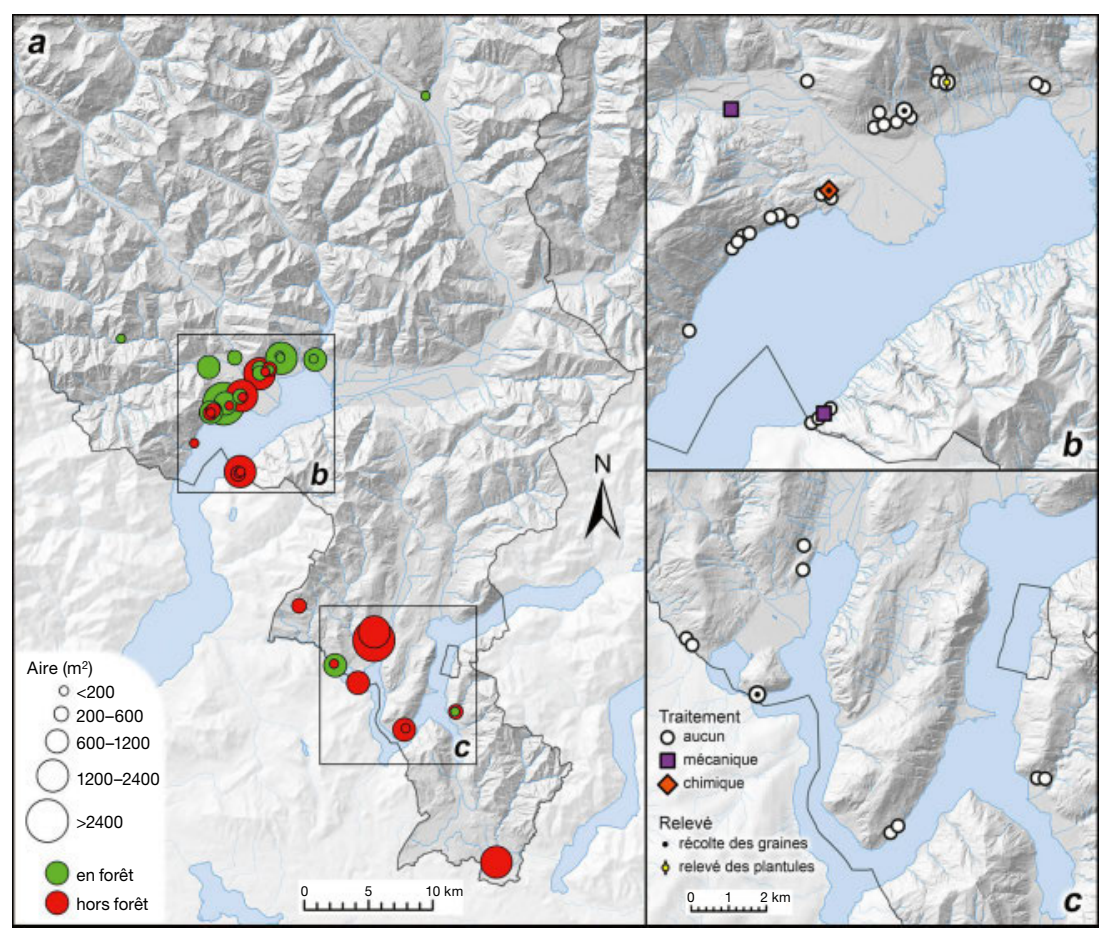

Fig. 3 Distribution des foyers de puéraire d'après I'inventaire 2017 et localisation des essais effectués. a) carte de l'ensemble de la zone; b) détail de la zone du Lago Maggiore; c) détail de la zone du Lago Ceresio. geurs et de maladies spécifiques et la croissance rapide de l'espèce ont favorisé son expansion rapide et en partie incontrôlée (Miller \& Edwards 1983). Ce comportement invasif a causé des dégâts significatifs en termes de biodiversité et de coûts directs et indirects à l'agriculture, à la filière du bois, et à la maintenance du réseau de distribution de l'électricité et des chemins de fer (Lindgren et al 2013). Ces coûts élevés n'ont de loin pas été contrebalancé par les bénéfices provenant de produits artisanaux (par ex. paniers, papier), fourragers et médicaux de la plante (Miller \& Edwards 1983). Classée officiellement comme plante envahissante dans les années 1970 (Alderman 2004), P. lobata est maintenant inclue dans les 100 espèces envahissantes les plus dangereuses au monde par le groupe des spécialistes des espèces envahissantes (Invasive Species Specialist Group ISSG 2017).

Différentes stratégies de lutte contre la puéraire sont mentionnées dans la littérature. Elles comprennent la lutte chimique (Miller \& Edwards 1983, Buholzer et al 2016), la lutte mécanique (Hardie \& Adams 2009) ${ }^{1}$ ou des méthodes combinées (Harrington et al 2003, Weaver \& Lyn 2007).

\section{Matériel et méthodes}

\section{Evaluation du potentiel invasif}

Afin de pouvoir évaluer la dynamique actuelle de colonisation et le potentiel invasif futur, nous avons demandé au cours de 2017 aux services forestiers cantonaux du Tessin et des Grisons de nous signaler les foyers supplémentaires non décrits par Morisoli et al (2013) et entretemps connus au sud des Alpes. Les foyers ainsi recensés ont été décrits en détail sur la base des paramètres suivants: emplacement (coordonnées; nature de propriété; géomorphologie; utilisation du sol; distances des maisons, des routes et des autres foyers), état végétatif (âge estimé; grandeur du foyer; pourcentage et hauteur de la partie grimpante; présence de fleurs, gousses et graines), impact existant en forêts, biotopes protégés et infrastructures, dégât dû à l'érosion et particularité du site (accessibilité au site; possibilité d'utilisation de produit de traitement chimique; urgence du traitement; nécessité d'une remise en état du site après traitement).

L'évaluation du potentiel de germination de la puéraire a été effectuée par un essai contrôlé en serre en 2013 et par des relevés de la germination in situ en 2017. Pour l'essai en serre, on a utilisé des graines provenant de trois sites (figure 3) et de deux années de récolte: Caslano (récoltées en octobre 2011 et en mai 2012), Orselina (récoltées en octobre 2011) et

\footnotetext{
1 HARDIE N, ADAMS L (2009) Surgical crown removal www.kokudzu.com/SurgicalRemoval.html (7.9.2017).
} 


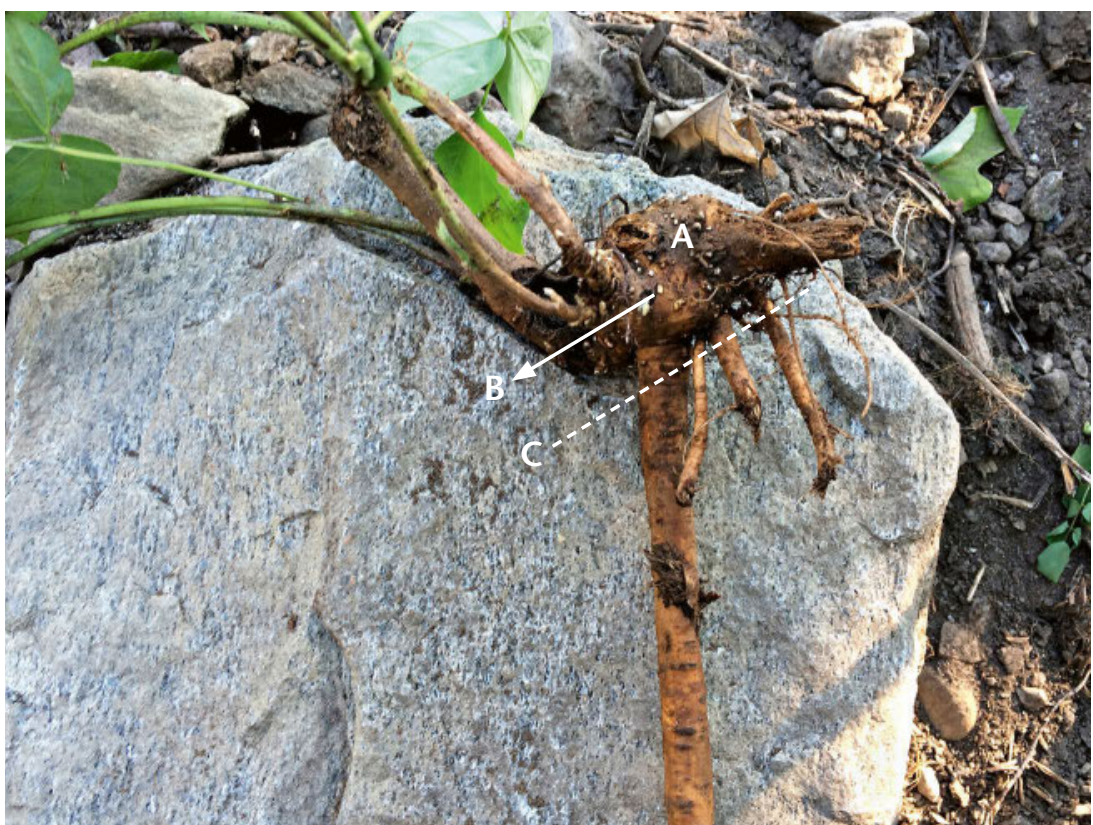

Fig. 4 Nœud enraciné et procédure de lutte mécanique. A) nœud enraciné; B) bourgeons végétatifs; C) ligne de coupe des racines du nœud. Photo: Romina Morisoli, 2016

Monte Verità (récoltées en avril 2012). Entre la récolte et le semis, les graines ont été stockées à $20^{\circ} \mathrm{C}$ et $40 \%$ d'humidité de l'air, sans subir de traitements supplémentaires particuliers (scarification, congélation, etc.). L'essai, mené en serre, comprenait deux régimes de température: serre chaude (température maximale moyenne de $26^{\circ} \mathrm{C}$ et température minimale moyenne de $20^{\circ} \mathrm{C}$ ) et serre froide (température maximale moyenne de $23^{\circ} \mathrm{C}$ et température minimale moyenne de $15^{\circ} \mathrm{C}$ ). Pour chaque régime de température on a utilisé trois répétitions de 50 graines pour chaque site et année de récolte. Les graines ont été semées le 31 mars 2013 en terrines avec du terreau Vitaflor Flox Alveo à $0.5-1 \mathrm{~cm}$ de profondeur. La germination a été contrôlée quotidiennement jusqu'à observer cinq jours consécutifs sans apparition de nouvelles plantules.

L'estimation du potentiel de reproduction sexuée à l'état naturel a été effectuée dans le foyer en forêt de Brione s/Minusio le 27 juillet 2017 (figure 3 , relevé des plantules). Une surface centrale de $324 \mathrm{~m}^{2}$ représentative de la structure de l'ensemble du foyer a été choisie et divisée par un maillage de $3 \times 3 \mathrm{~m}$. Une placette de $50 \times 50 \mathrm{~cm}\left(0.25 \mathrm{~m}^{2}\right)$ située sur chacun des 28 nœuds du maillage a été ensuite utilisée pour compter les plantules issues de la banque de semis naturels et pour vérifier la présence de débris de gousses. L'ouverture de la canopée a été estimée à l'aide des photos vers le ciel prises avec un téléphone portable (iPhone 5), en plaçant horizontalement l'appareil sur le sol au centre de chaque placette. La mesure a été calculée en pourcentage de pixels blancs, après transformation des images en noir et blanc avec la fonction «threshold» du package imager (version 0.40) du logiciel statistique R-Cran (R Core Team 2017).

\section{Evaluation des stratégies de lutte}

Dans cette phase d'étude préliminaire, la compatibilité et l'aptitude des méthodes de lutte mécanique et chimique ont été testées pour les lieux de la zone d'étude.

L'essai de lutte chimique a été effectué en 2012 dans une parcelle hors forêt du Parc du Monte Verità à Ascona (figure 3), dans les mêmes conditions écologiques et climatiques, mais avec d'autres principes actifs que ceux utilisés par Buholzer et al (2016) dans leur essai de 2007. Les variantes de traitement étaient: témoin, Garlon ${ }^{\circledR} 120$ (Triclopyr 120 g/l) appliqué à 15.6 1/ha, Effigo ${ }^{\circledR}$ (Clopyralide $267 \mathrm{~g} / 1+$ Piclorame $67 \mathrm{~g} / \mathrm{l}$ ) appliqué à 4.2 1/ha et Effigo ${ }^{\circledR}$ (Clopyralide $267 \mathrm{~g} / 1$ + Piclorame $67 \mathrm{~g} / \mathrm{l}$ ) appliqué à 8.4 1/ha. L'essai a été répliqué trois fois sur des parcelles de $4 \times 2$ m divisées entre elles par une coupe de toutes les lianes aériennes et celles à $5 \mathrm{~cm}$ sous terre. Avant l'application des herbicides, un nettoyage de toute la végétation présente dans les parcelles a été effectué en période de repos végétatif (automne/printemps) à l'aide d'une débroussailleuse. La première application a eu lieu le 20 juin 2012 au moyen d'un pulvérisateur à dos et en utilisant une quantité de bouillie de 1042 1/ha. La deuxième année, les traitements ont été répétés uniquement dans les parcelles avec présence de puéraire le 26 juin 2013. L'efficacité du traitement a été calculée en relevant pendant la période de végétation les paramètres suivants: mortalité des plantes, nombre de plantes et nœuds enracinés vivants par $\mathrm{m}^{2}$, longueur, hauteur et vitalité des plantes, degré de couverture de la canopée, largeur de la feuille centrale, nombre de feuilles par $\mathrm{m}^{2}$ et hauteur de la végétation. Ces relevés ont été effectués avant l'application des traitements chimiques le 19 juin 2012 et le 19 juin 2013, et à la fin de l'essai le 18 juillet 2014 .

Vue la défaillance de l'approche mécanique basée sur une fauche systématique pendant trois années pour éliminer la puéraire (Buholzer et al 2016), notre essai d'éradication mécanique a été inspiré par la technique de la coupe du collet proposée par Hardie \& Adams (2009) et consistant à couper les racines à quelques centimètres au-dessous des nœuds enracinés, de façon à éliminer tous les bourgeons végétatifs capables de rejeter (figure 4). Débuté en 2016, l'essai s'est déroulé sur deux sites fortement infestés: à Dirinella (commune de Gambarogno) et Zandone (commune de Losone; figure 3). Sur chaque site, quatre parcelles de $5 \times 3 \mathrm{~m}$ ont été d'abord libérés à la main en pleine saison végétative de toute végétation de puéraire présente pour assurer l'accessibilité et pour permettre d'identifier les nœuds enracinés. Le reste du foyer a été laissé intact en guise de contrôle. Le 3 août 2016, lorsque les nouvelles repousses ont atteint $50 \mathrm{~cm}$ environ, tous les nœuds repoussant ont été éliminés par une coupe des racines sous le noud avec une scie repliable à coupe 
tirante. Pour éliminer les nœuds encore actifs, l'opération a été répétée le 8 septembre 2016 et au cours de la saison 2017 une fois par mois à partir du 23 mai. A chaque passage, la couverture totale de la puéraire, le nombre de nœuds coupés, le diamètre de la racine la plus grosse pour chaque nœud, la profondeur du nœud et le temps total d'exécution ont été notés. Le matériel issu de la coupe a été laissé sur place, en prenant soin que les lianes et les parties ligneuses dessèchent au soleil le plus vite possible.
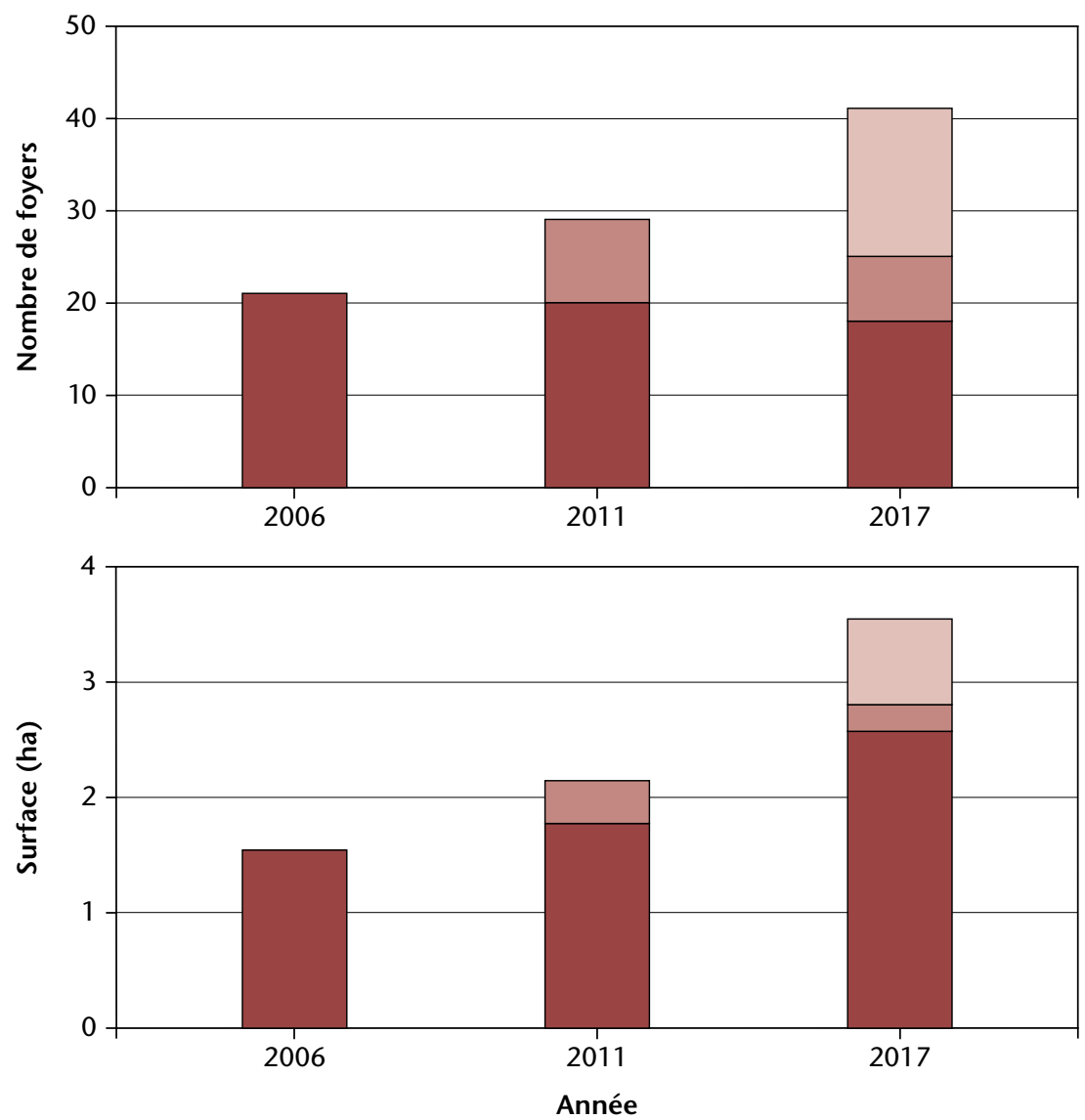

$\square$ Inventorié en $2006 \quad \square$ Nouveau en $2011 \quad \square$ Nouveau en 2017

Fig. 5 Evolution de la présence de puéraire au cours des inventaires. La diminution des foyers éradiqués entre un inventaire et l'autre est visible seulement dans le nombre de foyers.

\begin{tabular}{|c|c|c|c|c|c|c|}
\hline & \multicolumn{3}{|c|}{ Inventaires } & \multicolumn{3}{|c|}{ Evolution } \\
\hline & 2006 & 2011 & 2017 & 2006-2011 & 2011-2017 & 2006-2017 \\
\hline \multicolumn{7}{|c|}{ Nombre de foyers } \\
\hline En forêt & 12 & 16 & 25 & 4 & 9 & 13 \\
\hline Hors forêt & 9 & 13 & 16 & 4 & 3 & 7 \\
\hline Eradications & & & & 1 & 4 & 5 \\
\hline Total & 21 & 29 & 41 & 8 & 12 & 20 \\
\hline \multicolumn{7}{|c|}{ Surface couverte $\left(\mathrm{m}^{2}\right)$} \\
\hline Total & 15290 & 21254 & 35141 & 5964 & 13887 & 19851 \\
\hline En forêt & 11270 & 15180 & 26872 & 3910 & 11692 & 15602 \\
\hline Hors forêt & 4020 & 6074 & 8269 & 2054 & 2195 & 4249 \\
\hline Eradications & & & & 50 & 107 & 157 \\
\hline
\end{tabular}

Tab. 1 Nombre de foyers et surface couverte d'après les différents inventaires de puéraire.

\section{Résultats}

Situation actuelle et dynamique d'expansion

A ce jour (novembre 2017), 41 foyers de puéraire sont signalés en Suisse italienne, couvrant une surface totale de $35141 \mathrm{~m}^{2}$. La plupart des populations se trouvent dans les districts de Locarno et Lugano, près des rives des deux lacs (figure 3). 25 foyers se situent en zone forestière et 16 dans des friches et à proximité de structures anthropiques (tableau 1). Les 18 foyers historiques signalés par Pron (2006) et toujours présents dans l'inventaire de 2017 couvrent aujourd'hui une surface de $25492 \mathrm{~m}^{2}$ avec un accroissement total de $68 \%$ en onze ans, avec une pointe de $86 \%$ pour les foyers situés en forêt (données non présentées). En général, la puéraire montre une dynamique d'expansion (figure 5) avec seulement cinq foyers complètement éradiqués depuis 2006, ceci pour une surface négligeable de $157 \mathrm{~m}^{2}$ (tableau 1).

En forêt ou en présence d'infrastructures permettant à la puéraire de pousser verticalement, elle développe un recouvrement total, soutenu par des lianes dont le diamètre peut atteindre $12 \mathrm{~cm}$ et qui produisent des graines dans les parties exposées au soleil.

\section{Capacité de germination}

Le taux moyen de germination est de $46.5 \%$, indépendamment des deux types de conditionnement utilisés (serre chaude, serre froide). Les deux régimes de température choisis se différencient seulement par une germination plus précoce d'une semaine dans la variante serre chaude (résultats non présentés). Les taux de germination se distinguent en revanche en fonction du site et de la période de récolte (figure 6), avec des pourcentages plus élevés pour les graines récoltées à Caslano en 2011 (moyenne $80.7 \%)$ et plus faibles au Monte Verità pour la récolte 2012 (moyenne 28.3\%).

La germination de la puéraire à l'état sauvage a été confirmée par les relevés dans le foyer de Brione s/Minusio avec une densité moyenne de 13.3 plantules au $\mathrm{m}^{2}$ et des pics de 48 plantules au $\mathrm{m}^{2}$ en fonction de la chute des graines et de la disponibilité de la lumière au sol (figure 7). Au cours de l'été 2017, l'observation sur d'autres sites a révélé des cas de présence de jeunes plantules issues de germinations de graines. Ceci même sur le site du Monte Verità, où les vieilles lianes chargées en graines avaient été éliminées en 2012 suite à la préparation d'un essai de lutte chimique.

\section{Stratégies de lutte}

Les deux produits Garlon ${ }^{\circledR} 120$ appliqué à $15.6 \mathrm{l} /$ ha et $\mathrm{Effigo}^{\circledR}$ appliqué à $8.4 \mathrm{l} /$ ha ont montré une efficacité de $100 \%$ dès la première application. L'efficacité du traitement Effigo ${ }^{\circledR}$ appliqué à 4.2 1/ha 


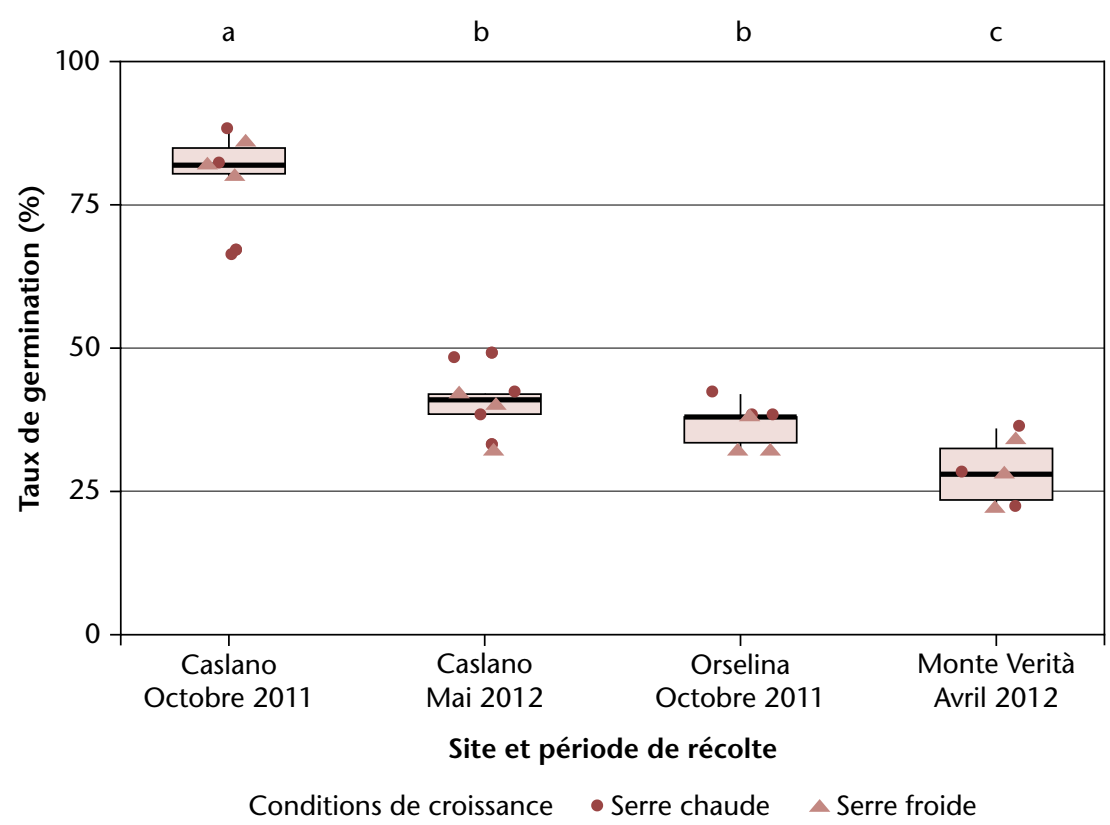

Fig. 6 Taux de germination issus de l'épreuve en serre en fonction du site et de la période de récolte. Chaque point représente une répétition de l'essai en serre chaude (cercles) ou froide (triangle). Les lettres $a$, $b$ et c représentent des situations statistiquement différentes.

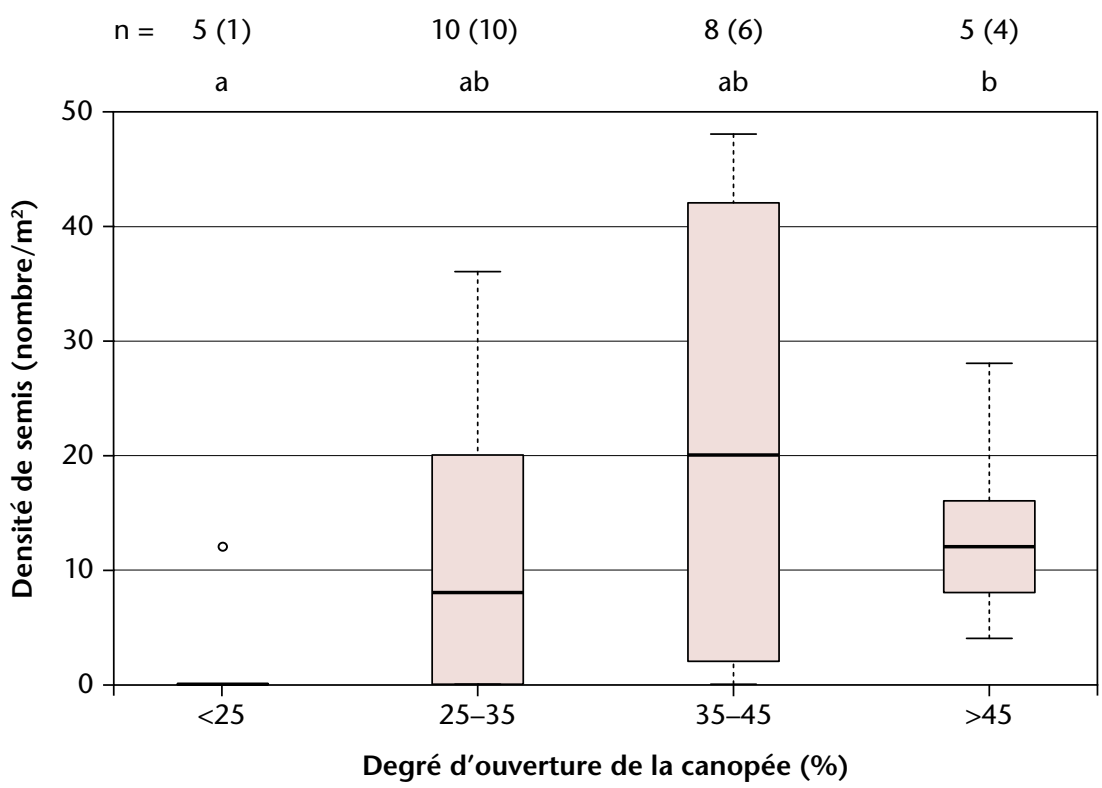

Fig. 7 Densité de plantules issues de la banque de graines du sol à l'état naturel en fonction du degré d'ouverture de la canopée. $n$ représente le nombre de relevés avec entre parenthèses le nombre de placettes avec présence de résidus de gousses. Les lettres a et $b$ représentent des situations statistiquement différentes $(p \leq 0.05)$ d'après le test de la somme des rangs de Wilcoxon-Mann-Whitney.

s'est révélée légèrement inférieure et ne garantit donc pas la complète élimination de la puéraire, même après deux ans de traitements (figure 8 ).

La figure 9 montre les effets de la lutte mécanique, par la réduction progressive du nombre de plantes par $\mathrm{m}^{2}$, qui s'approche rapidement d'une efficacité totale. L'investissement en temps est maximal lors de la première intervention et se réduit drastiquement pendant les visites de contrôle et l'élimination des nœuds enracinés résiduels.

\section{Discussion et stratégie de gestion}

L'évaluation de la situation actuelle et du potentiel d'invasion de la puéraire au sud des Alpes suisses nous indique l'existence d'une population spontanée et en constante augmentation. Pour le moment, cette expansion ne s'avère pas encore très explosive: les nouveaux foyers découverts depuis 2011 sont limités et certains n'ont probablement pas été ni vus, ni signalés dans les inventaires précédents. Une datation a posteriori de l'âge précis des foyers nouvellement découverts est assez difficile.

L'augmentation significative des surfaces des foyers historiques confirme en tous cas la propension de l'espèce à se diffuser localement surtout par voie végétative. Cette évolution est particulièrement critique en forêt et dans les zones protégées où l'accumulation de biomasse de puéraire représente un problème non seulement pour la biodiversité, mais aussi pour les incendies de forêt. Les lianes grimpantes sur les arbres constituent en effet une dangereuse continuité de matière combustible entre le sol et la canopée.

Néanmoins, les résultats de l'essai de germination et la détection de plantules issues de graines à l'état naturel nous indiquent un potentiel de production de banque de graines dans les foyers existants, d'autant plus que la probable survie pluriannuelle des graines dans le terrain (comme cela a été le cas au Monte Verità) signale un risque accru de colonisation d'espaces naturels par reproduction sexuée.

La population de puéraire a donc franchi la phase de naturalisation et peut potentiellement bientôt atteindre la phase de rapide expansion. Dans ce sens, le potentiel de dispersion des semis par les animaux va être décisif; un aspect encore très peu connu et qui mérite une attention particulière dans les recherches futures.

Le taux d'éradication très bas (seulement cinq foyers entre 2006 et 2017 pour une surface totale négligeable de $157 \mathrm{~m}^{2}$ ) et l'augmentation du nombre de foyers existants montrent l'inefficacité de la lutte occasionnelle, d'autant plus que les réactions des propriétaires des fonds concernés contactés pendant les relevés des foyers de puéraire nous ont montré un manque d'information sur le réel potentiel envahissant de l'espèce. Les essais de luttes conduits sont pourtant très prometteurs et démontrent que des interventions systématiques pourraient permettre d'éradiquer cette espèce, même en milieu forestier ou dans des biotopes protégés.

Sur la base de cette analyse et des résultats obtenus, l'objectif stratégique d'une éradication totale à moyen terme (5-8 ans) de l'espèce du sud des Alpes suisses nous semble tout à fait raisonnable. Les mesures concrètes énumérées ci-après sont envisageables. Tout d'abord, l'intensification de l'information au public et aux professionnels du secteur et la 


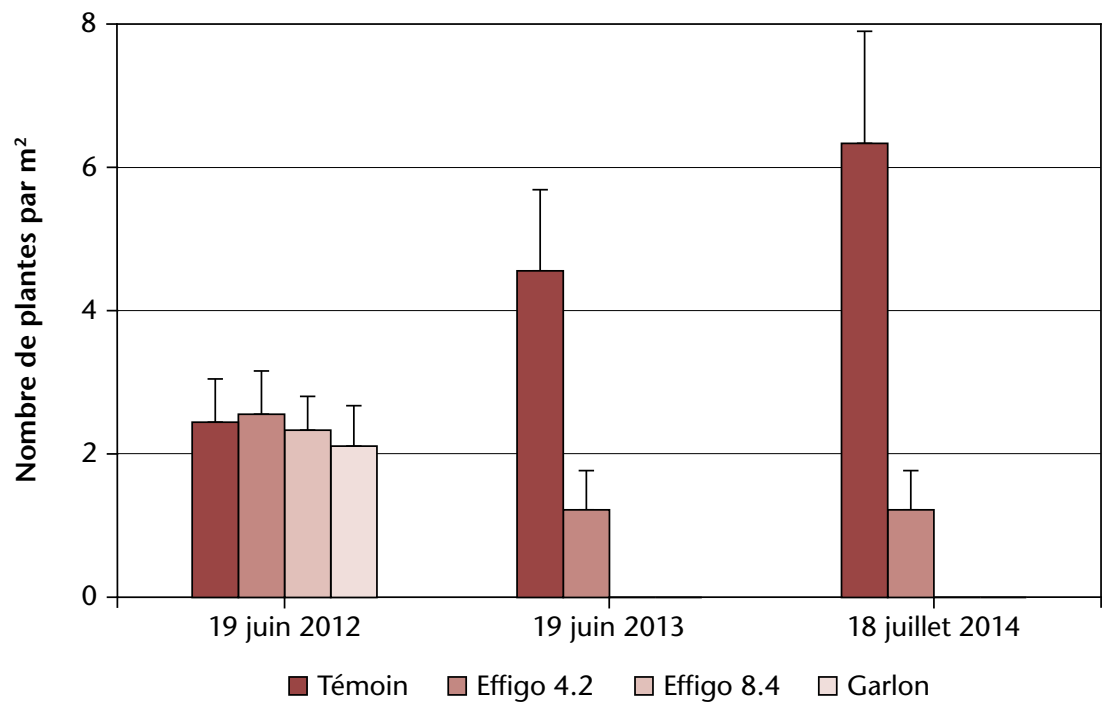

Fig. 8 Effets des traitements chimiques sur la puéraire.
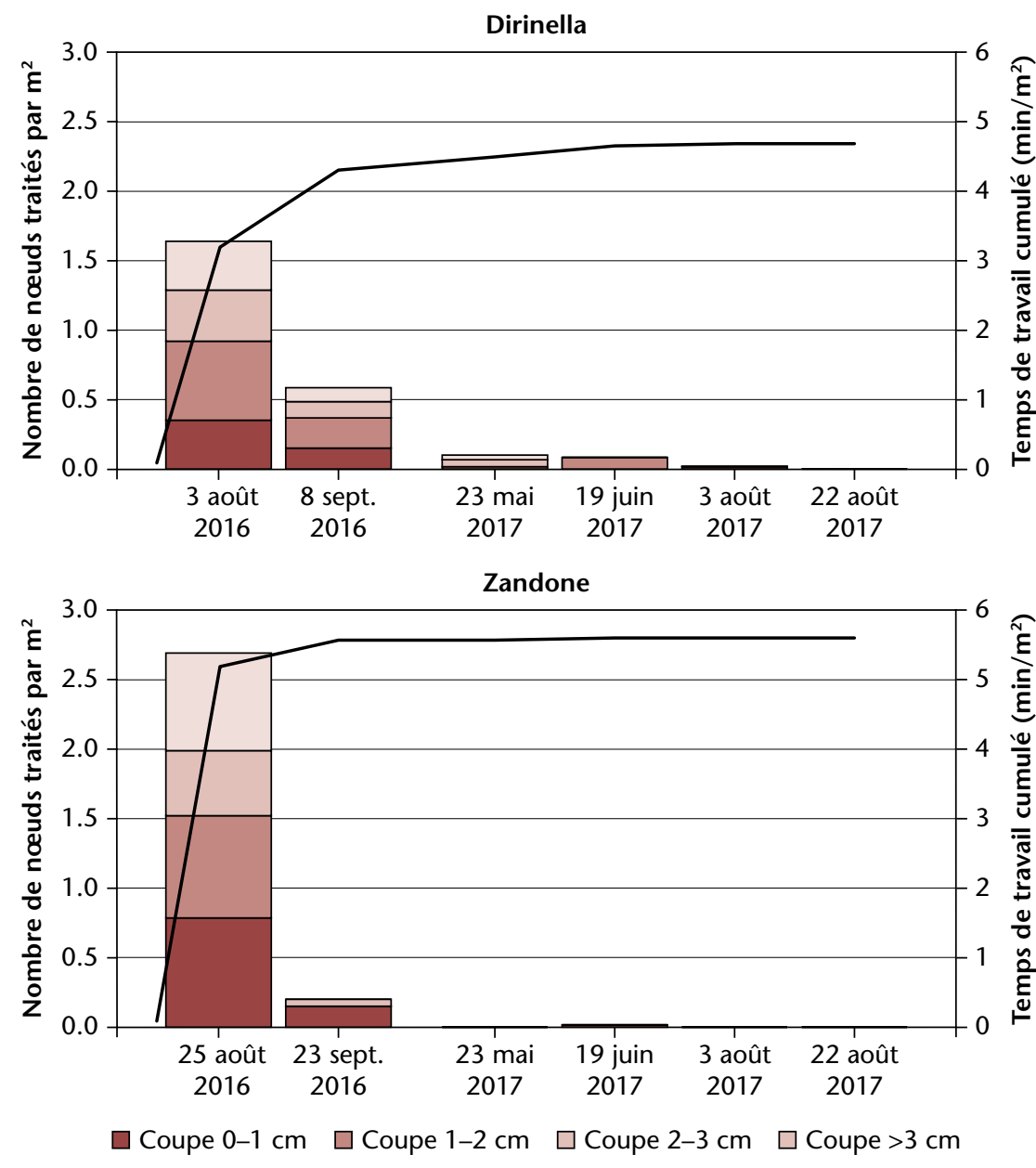

Fig. 9 Nombre de coupes des racines du nœud et temps cumulatif des essais de lutte mécanique. Les catégories de diamètre $(0-1 \mathrm{~cm}$ jusqu'à $>3 \mathrm{~cm})$ correspondent à la plus grosse racine coupée dans chaque nœud traité; le temps de travail est calculé sur la moyenne effective de deux minutes par nœud issue des épreuves effectuées.

formation d'équipes de spécialistes pour la lutte chimique et mécanique. Il s'agit aussi d'organiser des moyens financiers nécessaires et de mettre en place une planification d'une action coordonnée de lutte systématique en fonction des priorités suivantes: 1imiter la diffusion en éradiquant les vieux foyers qui produisent déjà des graines; limiter l'impact sur la biodiversité en éradiquant les foyers menaçant des biotopes protégés; éliminer les foyers difficilement accessibles avant leur croissance explosive et enfin s'occuper des foyers restants. Il faut faire le choix du type de lutte en fonction de la typologie du foyer (lutte chimique ou lutte mécanique), envisager des mesures de restauration et de gestion durable pour les surfaces traitées des foyers les plus étendus, en collaboration avec les services forestiers ou de la protection de la nature en fonction du type de site, effectuer la surveillance des sites traités pendant plusieurs années du fait de la capacité de recolonisation à partir de la banque de graines et, finalement, enregistrer et contrôler les nouveaux signalements de foyers en tant qu'activité permanente.

\section{Conclusions}

La puéraire au sud des Alpes suisses est un cas d'école. Il démontre qu'une évaluation précoce du problème, combinée à un approfondissement des connaissances sur l'écologie de l'espèce et la mise au point de méthodes de lutte efficaces pourrait conduire à un plan d'éradication coordonné. Ceci avec des bonnes chances de succès et à des coûts raisonnables.

Soumis: 20 septembre 2017, accepté (avec comité de lecture): 22 novembre 2017

\section{Références}

ALDERMAN DH (2004) Channing cope and the making of a miracle vine. Geogr Rev 94: 157-177.

BISCHOFF W, CUENI J, PEISL-GAILLET Y, KOLLY D (2014) Bekämpfung invasiver Neophyten: beschränkte Mittel zielgerichtet einsetzen (Essay). Schweiz Z Forstwes 165: 132-139. doi: 10.3188/szf.2014.0132

BUHOLZER S, PRON S, GIGON A (2016) Die Bekämpfung von Kudzu (Pueraria lobata): Die im Tessin invasive asiatische Liane lässt sich mit Mahd und Herbiziden eindämmen. Agroscope Transfer 116: 1-8.

COINER HA (2012) The role of low temperatures in determining the northern range limit of Kudzu (Pueraria montana var. 10bata), an invasive vine in North America. Toronto: Univ Toronto, Dep Ecology Evolutionary Biology, PhD Thesis. 234 p.

FOLLAK S (2011) Potential distribution and environmental threat of Pueraria lobata. Open Life Sci 6: 457-469.

HARRINGTON TB, RADER-DIXON LT, TAYLOR JR JW (2003) Kudzu (Pueraria montana) community responses to herbicides, burning and high-density Loblolly Pine. Weed Sci 51: 965-974.

HULME PE (2009) Trade, transport and trouble: managing invasive species pathways in an era of globalization. J Appl Ecol 46: 10-18.

INFO FLORA (2013) Fiche d'information sur la néophyte: Pueraria lobata. Berne: Info Flora. 5p. www.infoflora.ch/assets/content/ documents/neophytes/inva_puer_lob_f.pdf (27.3.2017)

KUEFFER C, BUGMANN H, CONEDERA M (2014) Invasive Neobiota im Wald: Konzepte und wissenschaftliche Grundlagen. Schweiz Z Forstwes 165: 124-131. doi: 10.3188/szf.2014.0124 
LINDGREN CJ, CASTRO KL, COINER HA, NURSE RE, DARBYSHIRE

SJ (2013) The biology of invasive alien plants in Canada:

12. Pueraria montana var. lobata (Willd.) Sanjappa \& Predeep. Can J Plant Sci 93: 71-95.

MESEINBURG MJ, FOX AM (2002) What role do birds play in dispersal of invasive plants? Wildland Weeds 5: 8-14.

MILLER J H, EDWARDS B (1983) Kudzu: Where did it come from? And how can we stop it? South J Appl For 7: 165-169.

MITICH L W (2000) Kudzu (Pueraria lobata [Willd.] Ohwi). Weed Techn 14: 231-235.

MORISOLI R, BERTOSSA M, ROSSINELLI M (2013) Pueraria lobata (Willd.) Ohwi nella Svizzera italiana: presenza sul territorio e potenziale invasivo. Boll Soc ticinese sci naturali 101: 93-97.

PRON S (2006) Ecologia, distribuzione e valutazione della liana esotica: Pueraria lobata (Willd.) Ohwi, Fabaceae, in Ticino. Zürich: Eidg Techn Hochschule, Dep Umweltsystemwissenschaften, MSc Thesis. $101 \mathrm{p}$.

R CORE TEAM (2017) R: A language and environment for statistical computing. www.R-project.org/ (27.11.2017).

\section{Strategie zur Bekämpfung eines invasiven Neophyten am Beispiel des Kudzu}

Kudzu (Kopoubohne; Pueraria lobata) ist eine aus Ostasien stammende Liane, die unter optimalen Bedingungen $30 \mathrm{~cm}$ pro Tag wachsen kann. In der Schweiz ist die Art auf der Schwarzen Liste der invasiven Neophyten aufgeführt. Zurzeit sind in der Südschweiz 41 Herde mit einer Gesamtfläche von $35141 \mathrm{~m}^{2}$ bekannt. In den letzten sechs Jahren hat die Fläche der Herde um 45\% zugenommen, 16 neue Kudzu-Standorte wurden entdeckt und vier wurden getilgt. Eine spontane Keimungsaktivität im Freien wurde festgestellt. Kudzu hat also die Phase der Etablierung hinter sich gelassen und droht nun in die Phase der schnellen Ausbreitung überzutreten. Entsprechend empfiehlt es sich, nun eine Strategie zu verfolgen, die die Art mittelfristig komplett tilgt. Vorversuche haben die Wirksamkeit chemischer Bekämpfungsmethoden bestätigt und es erlaubt, eine mechanische Bekämpfungsmethode (Abschneiden der Wurzeln von der Knolle) für den Einsatz im Wald und auf Naturschutzflächen zu testen. Die vorliegende Untersuchung zum Kudzu darf als Idealbeispiel für das Zusammenspiel der Vertiefung des Wissens über die Ökologie einer Art und der frühzeitigen Entwicklung von Behandlungsstrategien betrachtet werden, wobei das Ziel die Entwicklung von Kontroll- bzw. Tilgungsmethoden zu angemessenen Kosten ist.
SUSKO D, MUELLER P, SPEARS J (2001) An evaluation of methods for breaking seed dormancy in kudzu (Pueraria lobata). Can J Bot 79: 197-203.

TSUGAWA H, KAYAMA R (1985) Studies on population structure of Kudzu Vine (Pueraria lobata Ohwi). Jap Soc Grassland Sci 31: 167-176.

VOIGT A (1920) Beiträge zur Floristik des Tessins. Zürich: Botanisches Museum Univ Zürich, Mitt 85: 332-357.

WEAVER MA, LYN ME (2007) Compatibility of a biological control agent with herbicides for control of invasive plant species. Nat Areas | 27: 264-268.

\section{Sources}

INVASIVE SPECIES SPECIALIST GROUP ISSG (2017) Global invasive species database. WWW.iucngisd.org (27.22.2017)

\section{Strategy to control an invasive neophyte: the study case of kudzu}

Kudzu (Pueraria lobata) is a vine native to East Asia that grows under optimal conditions up to $30 \mathrm{~cm}$ per day. In Switzerland, the species is found on the blacklist of the invasive neophytes. At present 41 spots covering $35141 \mathrm{~m}^{2}$ are known in southern Switzerland. Historical spots known since 2006 have extended their area by $45 \%$ in the last six years, whereas 16 new spots have been registered and four sites have been eradicated. Seeds produced by mature lianas revealed to be viable and able to germinate in the wild. Kudzu has thus passed the naturalization stage and may soon enter the phase of a possible rapid expansion, what suggests adopting a strategy of systematic eradication of the species in the mid-term. Preliminary tests allowed to confirm the efficiency of the chemical and the mechanical (surgical removing of root crowns) control methods, the last allowing the treatment of kudzu in forest and in protected areas as well. Kudzu represents thus a good example for how combining early detection, better understanding of the ecology, and the development of suitable eradication methods of the species may allow to control or even systematically eradicate a species at reasonable costs. 